ISSN 0103-9954

\title{
PROPRIEDADES FÍSICAS E MECÂNICAS DE PAINÉIS MDF DE DIFERENTES MASSAS ESPECÍFICAS E TEORES DE RESINA
}

\section{MECHANICAL AND PHYSICAL PROPERTIES OF MDF PANELS OF DIFFERENT DENSITIES AND RESIN CONTENT}

\author{
Jackson Roberto Eleotério $^{1}$ Mario Tomazello Filho ${ }^{2}$ Geraldo Bortoletto Júnior ${ }^{2}$
}

\begin{abstract}
RESUMO
Painéis MDF foram preparados, em escala laboratorial, com base em fibras de Pinus spp. com diferentes teores de resina $\left(6,8,10,12\right.$ e 14\%) e massas específicas $\left(600\right.$ a $\left.800 \mathrm{~kg} / \mathrm{m}^{3}\right)$ com o objetivo de avaliar suas propriedades físicas e mecânicas. Os efeitos da massa específica e do teor de resina, bem como sua interação foram avaliados tendo como base as propriedades físicas e mecânicas dos painéis MDF. As propriedades mecânicas avaliadas foram módulo de elasticidade e de ruptura e adesão interna, enquanto as propriedades físicas foram absorção de água e inchamento em espessura. Todas as variáveis se relacionaram significativamente com os fatores massa específica e teor de resina e a interação foi significativa a $1 \%$ apenas no inchamento em espessura. Foram propostos modelos para avaliar as propriedades físicas e mecânicas em razão da massa específica e do teor de resina.
\end{abstract}

Palavras-chave: painéis MDF, teor de resina, densidade, propriedades físicas e mecânicas.

\begin{abstract}
MDF Panelboards of Pinus spp. fibers were prepared in laboratorial scale, within a range of densities $(600-800 \mathrm{~kg} / \mathrm{m} 3)$ and urea-formaldehyde resin content $(6,8,10,12$ and 14 wt. \%), with the purpose of evaluating their mechanical and physical properties. The effect of density and resin content and their interactions were analyzed based on panel mechanical and physical properties. Mechanical properties evaluated were modulus of rupture and modulus of elasticity and internal bond, while physical properties studied were thickness swelling and water absorption. All the physical and mechanical properties of the MDF panels were significantly related to the resin content and density. Models have been proposed to evaluate the physical and mechanical variables of the MDF panels taking into account the average density and resin content.
\end{abstract}

Key words: MDF, resin content, density, physical and mechanical properties.

\section{INTRODUÇÃO}

Os painéis MDF (Medium Density Fiberboard) são produzidos com fibras celulósicas misturadas com resinas sintéticas e que, prensadas a quente pelo processo seco, resultam em

1. Engenheiro Florestal, M.Sc., Professor do Departamento de Engenharia Florestal, Fundação Universidade Regional de Blumenau, CEP 89012-900, Blumenau (SC).

2. Engenheiro Florestal, Dr., Professor do Departamento de Ciências Florestais, Escola Superior de Agricultura Luiz de Queiróz, Universidade de São Paulo, CEP 13418-900, Piracicaba (SP). 
painéis com ensidades de 500 a $800 \mathrm{~kg} / \mathrm{m}^{3}$ (MALONEY, 1996). Esses painéis apresentam vantagens, como a reciclagem e renovação da matéria-prima, imobilização do carbono em sua composição e menor demanda de energia para produção em relação ao aço, plástico, alumínio, etc. Outras vantagens são a alta relação entre resistência mecânica e massa específica, boa trabalhabilidade, homogeneidade, ausência de defeitos (nós, desvios de grã, medula, etc.), agregação de valor a materiais provenientes de desbastes ou resíduos do processamento, além da possibilidade de controle das suas propriedades pela manipulação da matéria-prima, do processo e do ambiente de uso.

Dentre os principais fatores que afetam as propriedades físicas e mecânicas dos painéis MDF citam-se a sua massa específica ou densidade (NELSON, 1973, WOODSON, 1976, MALONEY, 1989 e KELLY, 1977). A massa específica dos painéis MDF tem influência positiva sobre os módulos de elasticidade (MOE) e de ruptura (MOR), não há, no entanto, consenso com respeito ao seu efeito sobre o inchamento, absorção de água e adesão interna (NELSON, 1973, CHOW, 1976 e MALONEY, 1989).

Verifica-se um aumento dos valores das propriedades mecânicas e da estabilidade dimensional dos painéis com o aumento do teor de resina, independente do seu tipo (MALONEY, 1989, LABOSKY JÚNIOR. et al., 1993, PIZZI, 1994 e BENADUCE, 1998).

O presente trabalho tem como objetivo avaliar conjuntamente o efeito da variação na massa específica e no teor de resina, bem como a interação entre esses fatores, sobre algumas propriedades físicas e mecânicas de painéis MDF como, inchamento em espessura, absorção de água, adesão interna e módulo de ruptura e de elasticidade. Visa também a determinar se o aumento da massa específica, com conseqüente maior contato entre fibras, resulta em aumento da eficiência da resina.

\section{MATERIAL E MÉTODOS}

\section{Delineamento experimental}

Os fatores teor de resina em cinco níveis $(6,8,10,12$ e 14\%) e massa específica dos painéis, também em cinco níveis $\left(600,650,700,750\right.$ e $\left.800 \mathrm{~kg} / \mathrm{m}^{3}\right)$ foram combinados de maneira fatorial, resultando em 25 tratamentos, aplicados com dois repetições em painéis MDF experimentais.

Foram utilizados painéis experimentais quadrados, com arestas de $43 \mathrm{~cm} \mathrm{e} \mathrm{1,6} \mathrm{cm} \mathrm{de}$ espessura, totalizando um volume unitário de $2958,4 \mathrm{~cm}^{3}$.

\section{Insumos}

\section{Fibras}

Foram utilizadas fibras, provenientes de uma mistura, sem controle da proporção, de cavacos de madeira de Pinus oocarpa SHIEDE e Pinus caribaea MORELET var hondurensis BARR ET GOLF. Esses cavacos foram desfibrados, após a sua digestão em vapor saturado à pressão entre 0,6 e 0,8 MPa, em um refinador industrial Andritz Sprout-Bauer, modelo 54/58-1CP.

As fibras obtidas tinham comprimento médio de $2,74 \mathrm{~mm}$, com desvio padrão de $51,1 \%$; largura

Ciência Florestal, v. 10 , n. 2, 2000 
média de 4,56 mm, com desvio padrão de $22,8 \%$ e espessura de parede de $1,00 \mathrm{~mm}$, com desvio padrão de $32,0 \%$.

As fibras foram secadas em estufa até teor de umidade de 3\% (base úmida).

\section{Resina}

A resina utilizada foi do tipo uréia-formaldeído reforçada com melamina, fabricada pela Duratex S.A., e as suas características físico-químicas foram determinadas antes do uso, podendo ser observadas na Tabela 1. A quantidade de adesivo aplicada nos painéis foi um dos fatores estudados e variou de acordo com cada tratamento empregado.

TABELA 1: Características da resina UF utilizada.

\begin{tabular}{lcc}
\hline Variável & Condições experimentais & Valor médio \\
\hline Teor de Sólidos & 4 horas a $105^{\circ} \mathrm{C}$ & $66 \%$ \\
Viscosidade Brookfield & $25^{\circ} \mathrm{C}$ & $150 \mathrm{cP}$ \\
$\mathrm{pH}$ & $25^{\circ} \mathrm{C}$ & 8,8 \\
Gel-time & $98^{\circ} \mathrm{C}$ & $130 \mathrm{~s}$ \\
Massa Específica & $20^{\circ} \mathrm{C}$ & $1,29 \mathrm{~g} / \mathrm{cm}^{3}$ \\
\hline
\end{tabular}

\section{Catalisador}

Como catalisador da cura da resina foi utilizado sulfato de amônia comercial, com teor de sólidos de $20 \%$, na dosagem de $2,5 \%$ de sólidos de catalisador em relação ao teor de sólidos da resina.

\section{Emulsão de parafina}

Foi utilizada uma emulsão de parafina comercial, com $70 \%$ de teor de sólidos, na proporção de $1 \%$ de sólidos da emulsão em relação à massa das fibras anidras, com a finalidade de reduzir a higroscopicidade final dos painéis MDF.

\section{Fabricação dos painéis}

A massa de resina foi determinada com base nos tratamentos, sendo correspondente a 6,8 , 10, 12 ou 14\% da massa anidra de fibras, e misturada à emulsão de parafina, ao catalisador e ainda à água, esta última na quantidade necessária para uniformizar o teor de umidade do colchão em $10 \%$ (base úmida).

A mistura dessas substâncias foi pulverizada através de uma pistola a ar comprimido sobre as fibras em uma encoladeira de laboratório. Na massa de fibras depositada em um dos compartimentos da encoladeira foi pulverizada a resina, com a rotação das pás metálicas em torno de $115 \mathrm{rpm}$. Em seguida a massa de fibras foi transferida para outro compartimento da encoladeira, que sem encontrava equipado com uma tela metálica de malha de $12,7 \mathrm{~mm}$ na sua parte inferior, promovendo a desagregação dos grumos de fibras e sua passagem pela malha. Foram utilizadas apenas fibras que passaram por essa malha. 
Tendo o volume de cada painel constante, a variação da massa específica foi determinada pela variação na massa de fibras adicionada ao painel.

As fibras, na massa necessária para atingir a massa específica determinada para cada painel, foram dispostas em uma formadora de laboratório. Sobre o colchão de fibras formado, foi aplicada uma carga de, aproximadamente, $700 \mathrm{~N}$, para melhor acomodação antes da pré-prensagem.

Os colchões de fibras foram pré-prensados, à uma pressão de 1,14 MPa, sem transferência de calor, por $14 \mathrm{~s}$.

A prensagem, em prensa hidráulica, seguiu o ciclo apresentado na Figura 1, controlado por $\mathrm{CNC}$, com a temperatura dos pratos regulada em $195^{\circ} \mathrm{C}$.

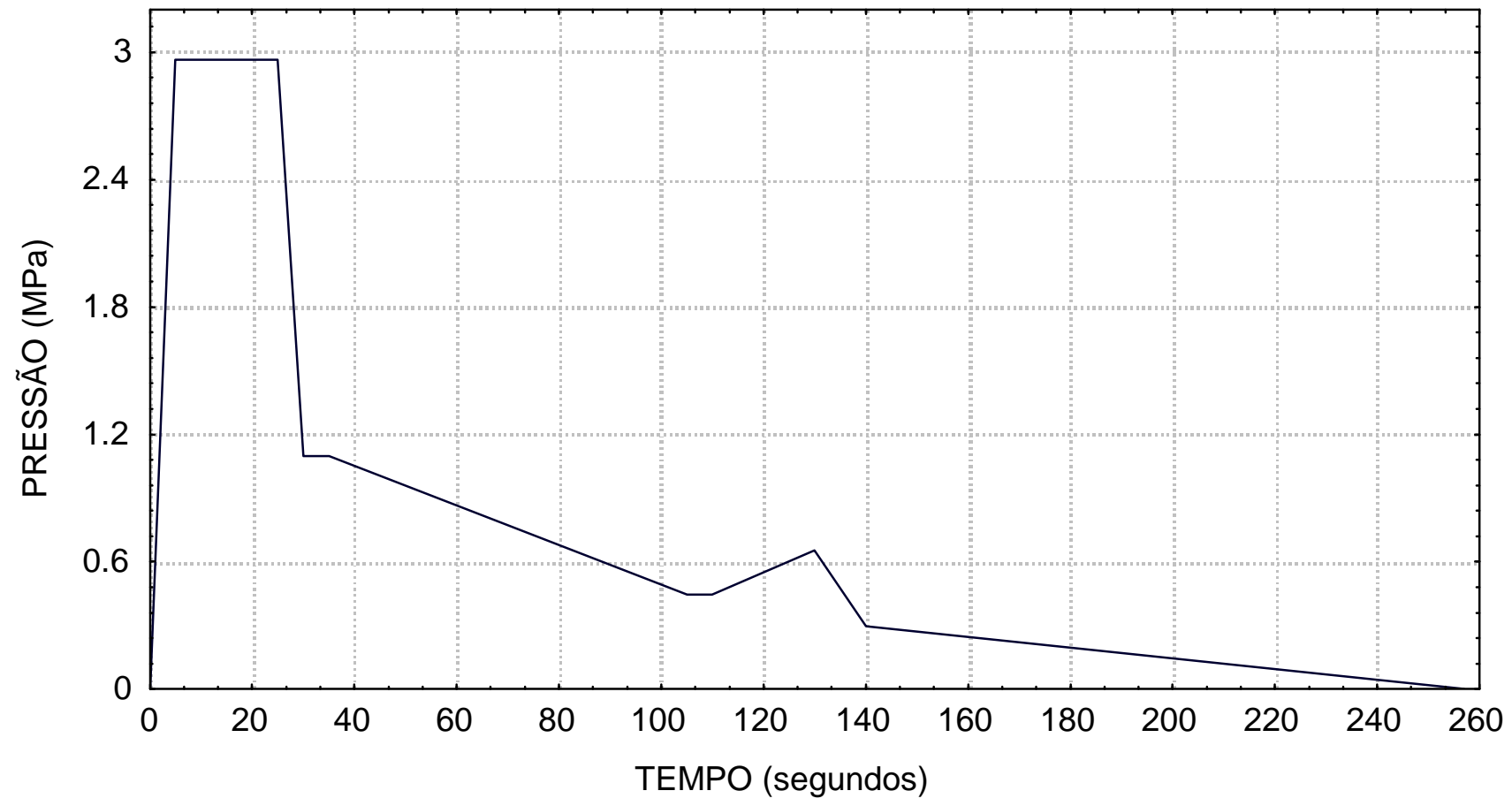

FIGURA 1: Ciclo de prensagem utilizado na confecção dos painéis MDF.

\section{Retirada dos corpos-de-prova e condicionamento}

Após resfriados à temperatura ambiente, os painéis MDF foram cortados, retirando-se dois corpos-de-prova para cada tipo de ensaio, conforme mostra a Figura 2. Os corpos-de-prova obtidos foram condicionados em câmara climatizada à temperatura de $20^{\circ} \mathrm{C}$ e umidade relativa de $65 \%$, até atingirem massa constante. Posteriormente, foram efetuados os ensaios e utilizou-se a média dos valores obtidos, dos dois corpos-de-prova de cada propriedade, considerada como representativa do valor da propriedade para o painel MDF. 


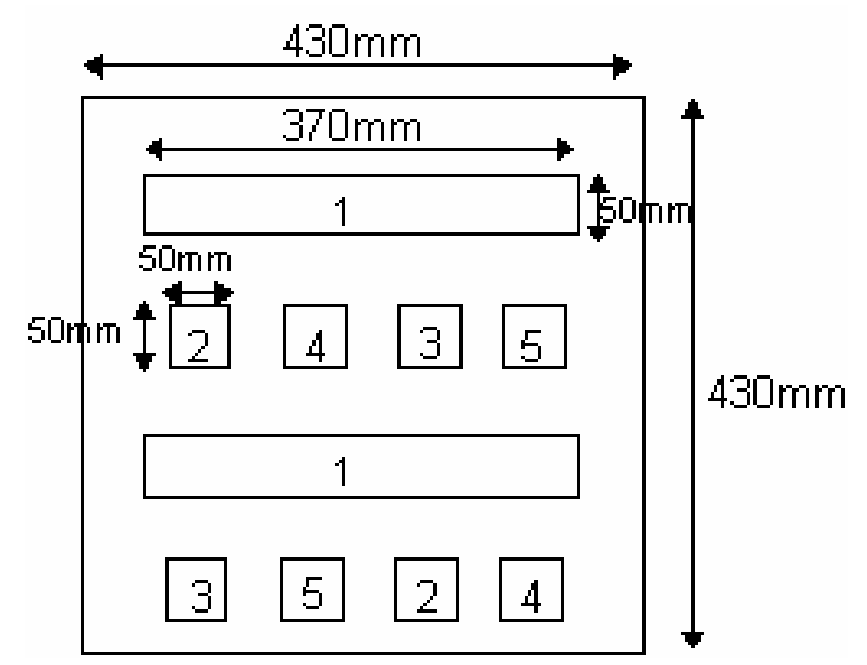

FIGURA 2: Representação de um painel MDF experimental e localização dos corpos-de-prova para os ensaios, sendo (1) para ensaio do módulo de elasticidade e de ruptura na flexão estática, (2) para a determinação da adesão interna, (3) para análise do inchamento em espessura e absorção de água, (4) para a determinação da massa específica e (5) para análise do perfil de densidade (não abordado neste trabalho).

\section{Ensaios}

\section{Módulo de ruptura e de elasticidade na flexão estática}

Os ensaios de módulo de ruptura e de elasticidade foram realizados segundo a norma EN3101993, que prescreve a utilização de corpos-de-prova com comprimento de vinte vezes a espessura mais $50 \mathrm{~mm}$. Nesse caso, 370 $\mathrm{mm}$ de comprimento por $50 \mathrm{~mm}$ de largura. A distância entre apoios é normatizada em vinte vezes a espessura e o incremento de carga ajustado para atingir a carga máxima em $60 \pm 30$ s.

\section{Inchamento em espessura e absorção de água}

Para os ensaios de inchamento em espessura utilizaram-se corpos-de-prova quadrados, com $50 \mathrm{~mm}$ de aresta, segundo a norma EN317-1993. Os corpos-de-prova foram imersos em água limpa, com pH $7 \pm 1$, temperatura de $20 \pm 1^{\circ} \mathrm{C}$, permanecendo cobertos por $25 \pm 5 \mathrm{~mm}$ de água durante 24 horas.

O inchamento em espessura foi determinado em relação à sua espessura inicial e a absorção de água determinada com base na sua massa inicial.

\section{Massa específica e adesão interna}

Para a determinação da massa específica foi aplicada a norma EN 323-1993, que prescreve a utilização de amostras quadradas, com $50 \mathrm{~mm}$ de aresta, condicionadas a $20^{\circ} \mathrm{C}$ e $65 \%$ de umidade relativa, determinando-se a relação entre a massa nestas condições e o volume exato. Para adesão 
interna foi utilizada norma EN 319-1993 que prescreve a utilização de amostras com 50mm de aresta, sendo determinada a resistência à tração perpendicular ao plano do painel.

\section{Análise estatística}

Inicialmente, as variáveis dependentes foram correlacionadas por meio do coeficiente de correlação de Pearson com os fatores massa específica e teor de resina. Em seguida, a significância da interação teor de resina e densidade foi testada para cada variável e foram gerados e ajustados modelos com o auxílio do procedimento Stepwise de regressão. Os modelos foram avaliados mediante análise do coeficiente de determinação $\left(\mathrm{R}^{2}\right)$, valor do teste $\mathrm{F}$, erro padrão da estimativa (Syx) e distribuição gráfica dos resíduos.

Sendo os dois fatores analisados, massa específica e teor de resina quantitativos, os modelos obtidos geravam superfícies de resposta. Para facilitar a visualização, essas superfícies foram projetadas num plano e traçadas isolinhas. Isolinhas correspondem a união de pontos com o mesmo valor da variável analisada.

\section{RESULTADOS E DISCUSSÃO}

\section{Painéis obtidos}

Os ensaios de massa específica de duas amostras em cada painel revelaram que a variação média intrapainel é de 5,2\%.

Já a variação entre a massa específica real dos painéis e a estipulada nos tratamentos foi maior, obtendo-se painéis com menor massa específica que o planejado e, assim, algumas faixas não foram representadas, como $800 \mathrm{~kg} / \mathrm{m}^{3}$.

Dois fatores que contribuíram para esse fato são o espalhamento das fibras durante a prensagem, determinando que a mesma massa de fibras se dispersasse por uma área maior que a planejada para o painel $(43 \times 43 \mathrm{~cm})$. Esse mesmo fato foi descrito por PUGEL et al. (1990). Outro fator determinante desse desvio, em relação do planejado, foi um inchamento do painel alguns décimos de milímetros após o alívio da pressão, em parte, resultado de um baixo teor de resina. Uma alternativa seria elevar o teor de umidade do colchão, tornando as fibras mais plásticas e reduzindo a tensão residual .

Apesar desse fato, a variação de massa específica, dentro de cada nível de resina, foi suficientemente ampla, permitindo a utilização desses painéis na análise. Os pontos de massa específica obtidos experimentalmente são apresentados, por nível de resina, nas Figuras 3 a 7.

\section{Correlação entre variáveis dependentes e independentes}

As correlações entre as variáveis dependentes analisadas e os fatores, massa específica e teor de resina em percentagem, expressas por meio do coeficiente de correlação linear de Pearson, são apresentados na Tabela 2. 
TABELA 2: Coeficientes de correlação linear de Pearson (\%) entre adesão interna, módulo de ruptura (MOR), módulo de elasticidade (MOE), inchamento em espessura e absorção de água e massa específica e teor de resina das chapas MDF.

\begin{tabular}{l|c|c|c|c|c}
\hline Fator & Ad. interna & MOR & MOE & Inchamento & Absorção \\
\hline Massa específica & 46,0 & 60,5 & 72,3 & $-49,9$ & $-57,2$ \\
Teor de Resina (\%) & 70,1 & 67,9 & 65,3 & $-69,7$ & $-64,5$ \\
\hline
\end{tabular}

Os módulos de ruptura e de elasticidade na flexão estática foram as duas variáveis que apresentaram maior correlação com a massa específica dos painéis.

Em relação ao teor de resina, expresso com base na massa anidra de fibras, a maior correlação encontrada refere-se a adesão interna, porém, em módulo, todas as variáveis analisadas apresentam correlação na mesma faixa.

Destacam-se as correlações negativas entre inchamento em espessura e absorção de água e as variáveis independentes, significando relações inversamente proporcionais.

\section{Interação entre massa específica e teor de resina}

Ao contrário do esperado, a interação entre os fatores massa específica e teor de resina não atuou significativamente sobre todas as variáveis. Nas variáveis relacionadas, a flexão estática à interação não foi significativa. A variável inchamento apresentou interação com a maior significância. Acreditava-se que uma maior massa específica promoveria maior contato entre fibras e assim maior eficiência no uso da resina, caracterizando uma interação e que tal fato seria significativo para todas as variáveis, o que não se confirmou para módulo de ruptura e de elasticidade.

TABELA 3: Interação entre os fatores densidade e teor de resina dos painéis MDF, expressa por meio do valor do teste $\mathrm{F}$.

\begin{tabular}{l|c|c|c|c|c}
\hline Fator & Ad. interna & MOE & MOR & Inchamento & Absorção \\
\hline $\begin{array}{l}\text { Massa Específica } x \\
\text { Teor relativo de resina }\end{array}$ & $4,46^{*}$ & $1,87^{\mathrm{ns}}$ & $2,65^{\mathrm{ns}}$ & $10,18^{* *}$ & $6,48^{*}$ \\
\hline
\end{tabular}

Em que: ** significativo a $1 \%$; * significativo a $5 \% ;{ }^{\text {ns }}$ não-significativo.

\section{Correlação entre variáveis dependentes}

A análise das correlações entre as variáveis dependentes, expressa pelo coeficiente de correlação de Pearson, é apresentada na Tabela 4.

Especialmente, as variáveis obtidas de um mesmo corpo-de-prova apresentaram maior coeficiente de correlação, ou seja, módulo de ruptura e de elasticidade, com correlação de $94 \%$ e inchamento em espessura e absorção de água, com 96,7\%.

A variável adesão interna apresentou maior correlação com os módulos de ruptura e de elasticidade em relação à correlação com o inchamento em espessura e a absorção de água.

Ciência Florestal, v. 10 , n. 2, 2000 
TABELA 4: Coeficientes de correlação linear de Pearson (\%) entre adesão interna, módulo de ruptura (MOR), módulo de elasticidade (MOE), inchamento em espessura e absorção de água dos painéis MDF.

\begin{tabular}{l|c|c|c|c|c}
\hline Fator & Ad. interna & MOR & MOE & Inchamento & Absorção \\
\hline Ad. Interna & 100,0 & & & \\
MOR & 89,6 & 100,0 & & & \\
MOE & 83,1 & 94,0 & 100,0 & & \\
Inchamento & $-66,8$ & $-75,7$ & $-78,8$ & 100,0 & \\
Absorção & $-67,9$ & $-79,6$ & $-83,0$ & 96,7 & 100,0 \\
\hline
\end{tabular}

\section{Módulo de ruptura}

O módulo de ruptura apresentou correlação de 60,5\% com a massa específica e 67,9\% com o teor de resina em percentagem (sólido de resina em relação à massa anidra de fibras).

Ajustando os valores desta variável em razão desses dois fatores anteriormente citados, foi obtido o modelo apresentado na equação 1.

$$
\operatorname{MOR}=b_{0}+b_{1} * M E+b_{2} * M E^{2}+b_{3} * \text { RESINA }
$$

Sendo: $\mathrm{MOR}=$ módulo de ruptura $(\mathrm{MPa})$;

$\mathrm{ME}=$ massa específica do painel $\left(\mathrm{kg} / \mathrm{m}^{3}\right)$;

RESINA = teor de resina $(\%)$;

$\mathrm{b}_{0}=+116,432383$;

$b_{1}=-0,395845$;

$\mathrm{b}_{2}=+3,4533 * 10^{-4}$;

$\mathrm{b}_{3}=+1,375850$.

O coeficiente de determinação deste modelo é de $71,6 \%$, valor do teste $\mathrm{F}$ de 38,66 , significativo a $1 \%$ e erro padrão da estimativa de 0,184 , bem como distribuição homogênea dos resíduos.

O modelo apresentado na equação 1 gera uma superfície de resposta que, projetada para um plano e dotada de isolinhas, ou seja, linhas que unem pontos de mesmo valor para a variável analisada, é apresentada na Figura 3.

Os teores de resina de 10 a 14\% apresentam apenas nove pontos pela coincidência de dois painéis com a mesma massa específica.

Constam no modelo os dois fatores analisados, teor de resina e massa específica, sugerindo efeito significativo e interativo sobre o módulo de ruptura.

O módulo de ruptura aumenta com o aumento do teor de resina e da massa específica. 


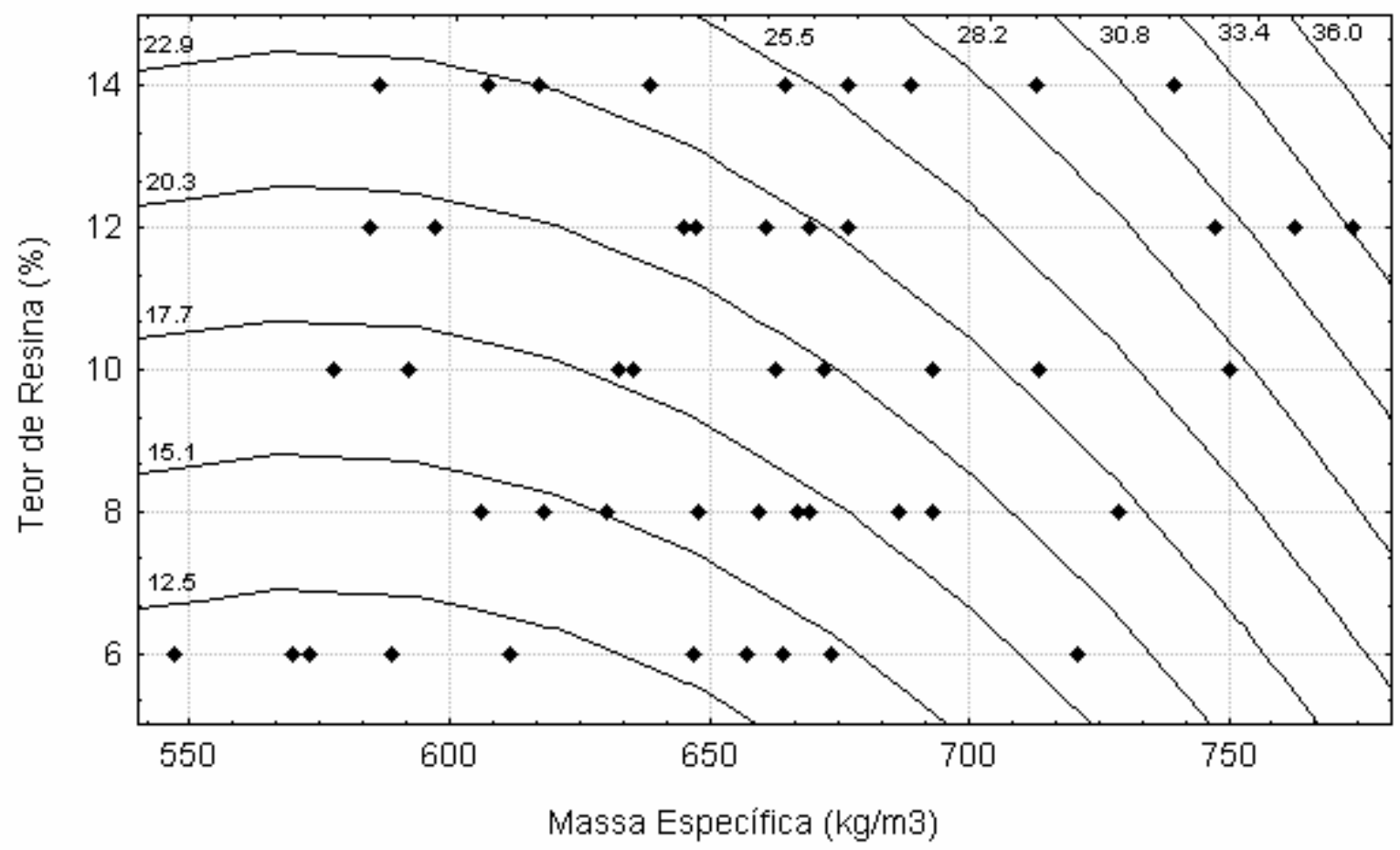

FIGURA 3: Isolinhas de módulo de ruptura (MPa) em razão da massa específica $\left(\mathrm{kg} / \mathrm{m}^{3}\right)$ e do teor de resina (\%) dos painéis MDF, com os losângulos representando os pontos obtidos experimentalmente.

\section{Módulo de elasticidade}

O módulo de elasticidade apresentou correlação de 72,3\% com a massa específica e de $65,3 \%$ com o teor de resina, sendo a única variável analisada que apresentou maior correlação com a massa específica do que com o teor de resina.

O modelo obtido para módulo de elasticidade em razão da massa específica e do teor de resina é apresentado na equação 2 .

$$
\mathrm{MOE}=\mathrm{b}_{0}+\mathrm{b}_{1} * \mathrm{ME}+\mathrm{b}_{2} * \mathrm{ME}^{2}+\mathrm{b}_{3} * \text { RESINA }
$$

Sendo: $\mathrm{MOE}=$ módulo de elasticidade $(\mathrm{GPa})$;

$\mathrm{ME}=$ massa específica do painel $\left(\mathrm{kg} / \mathrm{m}^{3}\right)$;

RESINA $=$ teor de resina $(\%)$

$\mathrm{b}_{0}=+7,035894$;

$b_{1}=-0,024088$;

$\mathrm{b}_{2}=+2,343 * 10^{-5}$;

$\mathrm{b}_{3}=+0,111354$.

A precisão do modelo pode ser comprovada pelo seu coeficiente de determinação, de 80,3\%, um valor do teste $\mathrm{F}$ de 62,46 , significativo a $1 \%$, e erro padrão da estimativa (Syx) de 0,110 ; bem 
como distribuição homogênea dos resíduos.

O modelo dado pela equação 2 é semelhante ao obtido para módulo de ruptura (eq. 1), diferindo somente nos coeficientes.

Observa-se, como na variável anteriormente analisada, a inclusão tanto da massa específica como do teor de resina, indicando atuação significativa desses dois fatores.

A projeção da superfície de resposta obtida da equação 2 é apresentada na Figura 4.

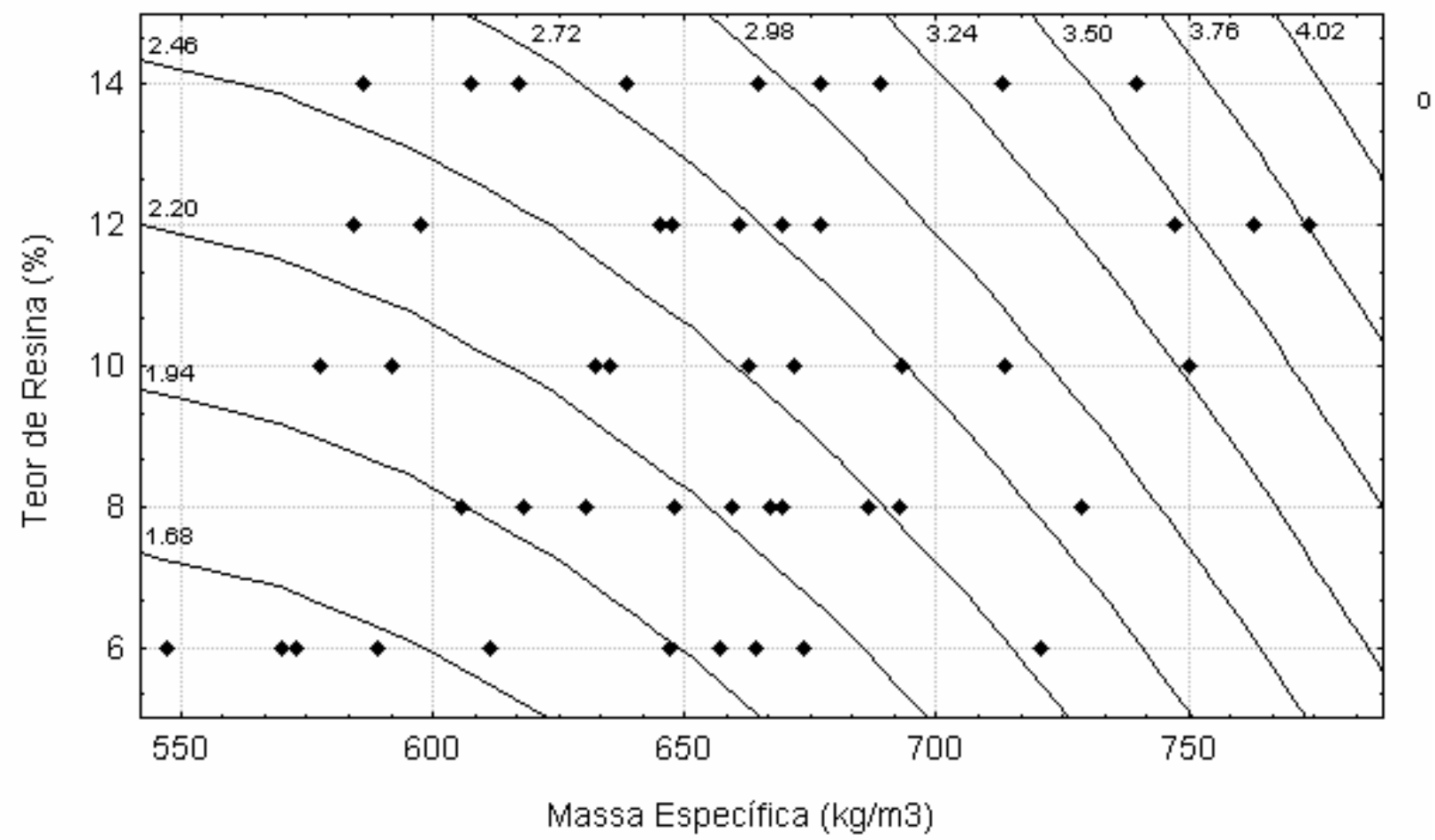

FIGURA 4: Isolinhas de módulo de elasticidade (GPa) em razão da massa específica $(\mathrm{kg} / \mathrm{m} 3)$ e do teor de resina (\%) dos painéis MDF, como os losângulos representando os pontos obtidos experimentalmente.

O comportamento do módulo de elasticidade na flexão foi semelhante ao do módulo de ruptura e cresce com o aumento da massa específica e do teor de resina.

\section{Inchamento em espessura}

A massa específica e o teor de resina apresentaram correlação de $-49,9$ e $-64,5 \%$ respectivamente, com o inchamento em espessura.

Um modelo para inchamento em espessura em razão da massa específica e do teor de resina é apresentado na equação 3 .

$$
\mathrm{INCH}=\mathrm{b}_{0}+\mathrm{b}_{1} * \mathrm{ME}^{-2}+\mathrm{b}_{2} * \text { RESINA }+\mathrm{b}_{3} * \mathrm{RESINA}^{-1}+\mathrm{b}_{4} * \mathrm{RESINA}^{-2}
$$


Sendo: $\mathrm{INCH}=$ inchamento em espessura (\%) após imersão em água;

$\mathrm{ME}=$ massa específica do painel $\left(\mathrm{kg} / \mathrm{m}^{3}\right)$;

RESINA $=$ teor de resina $(\%)$;

$\mathrm{b}_{0}=+275,267860$

b1 $=+4961621,375978$;

b2 $=-9,407572$;

b3 = -2642,690261;

b4 $=+8419,506187$.

O modelo apresentou coeficiente de determinação de 78,4\%, valor do teste $\mathrm{F}$ de 40,77 e erro padrão da estimativa (Syx) de 0,226; bem como distribuição homogênea dos resíduos. Figura 5 .

A projeção no plano da superfície de resposta obtida com a equação 3 é apresentada na

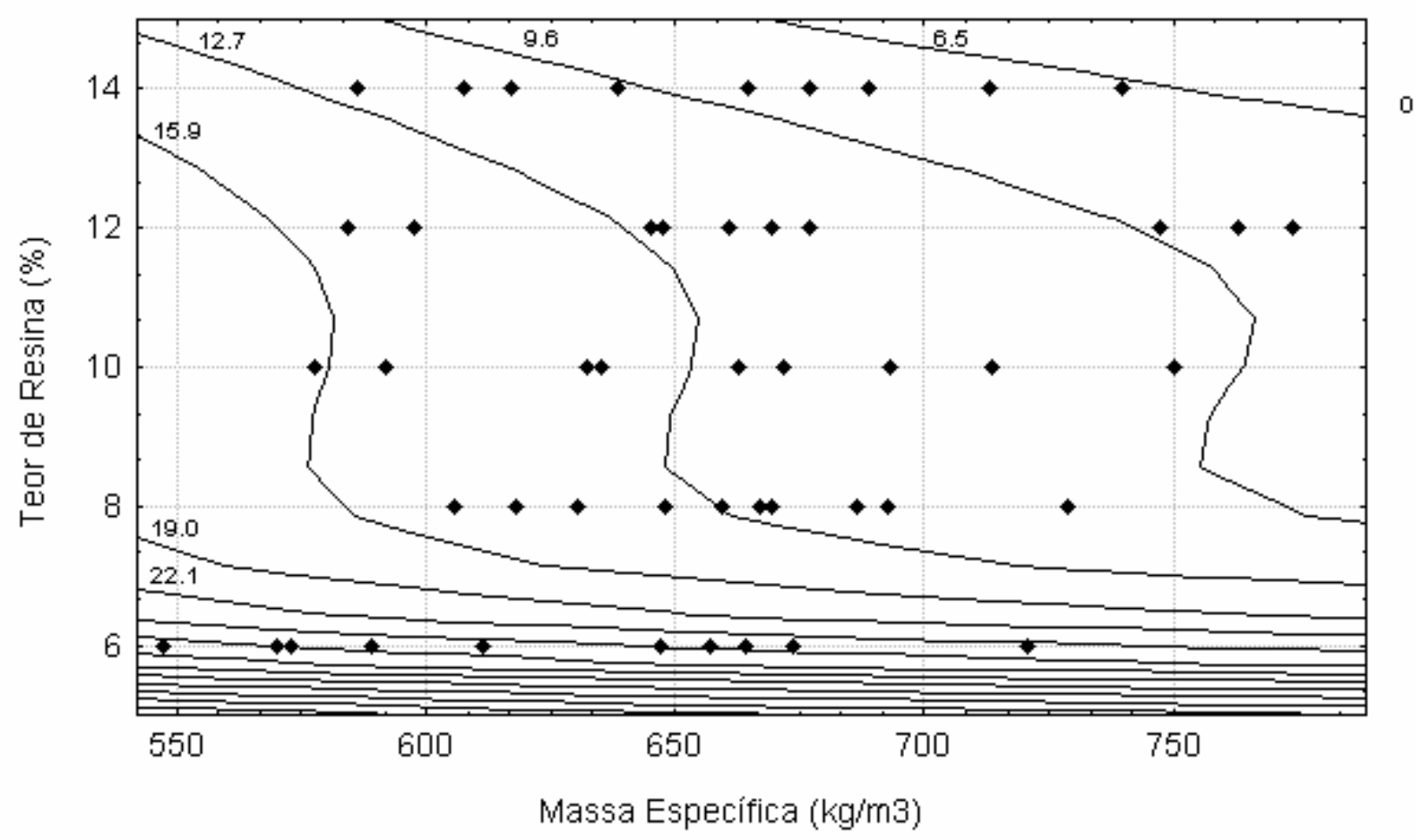

FIGURA 5: Isolinhas de inchamento em espessura (\%) em razão da massa específica $(\mathrm{kg} / \mathrm{m} 3)$ e do teor de resina (\%) dos painéis $\mathrm{MDF}$, com os losângulos representando os pontos obtidos experimentalmente.

Pela Figura 5, observa-se a maior influência do teor de resina. Ocorre especialmente uma redução no inchamento em espessura em painéis de 6 para $8 \%$ de teor de resina. Ao longo dos valores de massa específica essa variação é menos acentuada.

A influência da massa específica sobre o inchamento em espessura é diversa, pois 
MALONEY (1989) afirma que essa variável contribui para o inchamento em espessura, já SUCHSLAND e WOODSON (1987), CHOW (1976) e NELSON (1973) afirmam que não. Aparentemente ocorre um componente positivo da massa específica que é o maior contato entre fibras, aumentando a eficiência da colagem e um componente negativo determinado pela maior massa de fibras e conseqüente maior pressão de inchamento.

Segundo KELLY (1977) e CARLL (1996), o inchamento em espessura em painéis apresenta um componente que é a tensão residual de compressão, aliviada durante o umedecimento e irreversível.

\section{Absorção de água}

A absorção de água seguiu o mesmo comportamento do inchamento em espessura, tendo correlação com a massa específica de $-57,2 \%$ e com o teor de resina de $-64,5 \%$.

O modelo obtido para essa variável, em razão dos fatores analisados, é apresentado na equação 4.

$$
\text { ABSORC }=b_{0}+b_{1} * M E^{-2}+b_{2} * \operatorname{RESINA}+b_{3} * \operatorname{RESINA}^{-1}+b_{4} * \operatorname{RESINA}^{-2}
$$

Sendo: ABSORC = absorção de água (\% da massa inicial);

$\mathrm{ME}=$ massa específica do painel $\left(\mathrm{kg} / \mathrm{m}^{3}\right)$;

RESINA $=$ teor de resina $(\%)$;

$\mathrm{b}_{0}=+656,3372$;

b1 $=-0,097627$;

b2 $=-18,8734$;

b3 = -5508,9430;

b4 $=+17703,773910$.

O modelo apresentou coeficiente de determinação de 80,1\%, valor do teste $\mathrm{F}$ de 45,43 e erro padrão da estimativa (Syx) de 0,195; bem como distribuição homogênea dos resíduos.

6.

A projeção da superfície de resposta obtida, com base na equação 4, é apresentada na Figura

\section{Adesão interna}

A correlação entre massa específica e adesão interna foi de $46,0 \%$ e entre esta variável e teor de resina de 70,1\%. O modelo obtido (equação 5) incorpora de maneira significativa esses dois componentes, evidenciando sua influência sobre a adesão interna. 


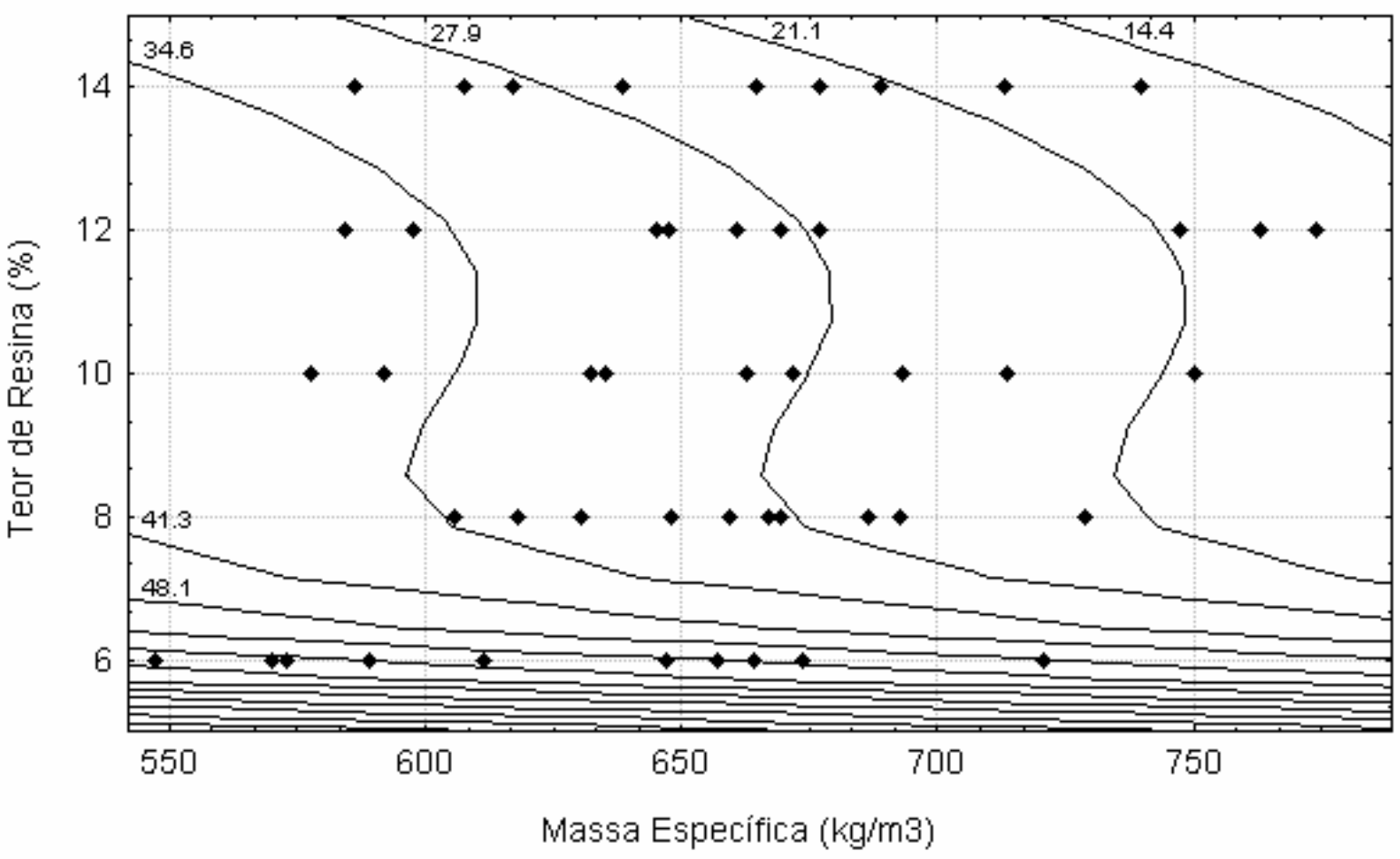

FIGURA 6: Isolinhas de absorção de água (\%) em razão da massa específica $(\mathrm{kg} / \mathrm{m} 3)$ e do teor de resina $(\%)$ dos painéis $\mathrm{MDF}$, com os losângulos representando os pontos obtidos experimentalmente.

$$
\mathrm{AI}=\mathrm{b}_{0}+\mathrm{b}_{1} * \mathrm{ME}+\mathrm{b}_{2} * \mathrm{ME}^{2}+\mathrm{b}_{3} * \text { RESINA }
$$

Sendo: $\mathrm{AI}=$ adesão interna $(\mathrm{MPa})$;

$\mathrm{ME}=$ massa específica do painel $\left(\mathrm{kg} / \mathrm{m}^{3}\right)$;

RESINA $=$ teor de resina $(\%)$;

$\mathrm{b}_{0}=+3,453229$;

$b_{1}=-0,011543$;

$\mathrm{b}_{2}=+9,38 * 10^{-6}$;

$\mathrm{b}_{3}=+0,031731$;

O modelo apresentou um coeficiente de determinação de $64,6 \%$, um valor do teste $\mathrm{F}$ de 28,04, significativo a $1 \%$, e erro padrão da estimativa (Syx) de 0,330, além de distribuição homogênea dos resíduos.

A projeção da superfície de resposta obtida da equação 5 é apresentada na Figura 7.

Observa-se um claro aumento da adesão interna com o aumento do teor de resina. Referente à massa específica, a relação não foi uniforme, até pouco mais de $600 \mathrm{~kg} / \mathrm{m}^{3}$, o aumento da massa específica determinou redução na adesão interna, porém acima desse valor, a massa específica 
contribui para o aumento da adesão interna. Pode ter havido uma concentração de painéis com menor consolidação nessa faixa de massa específica.

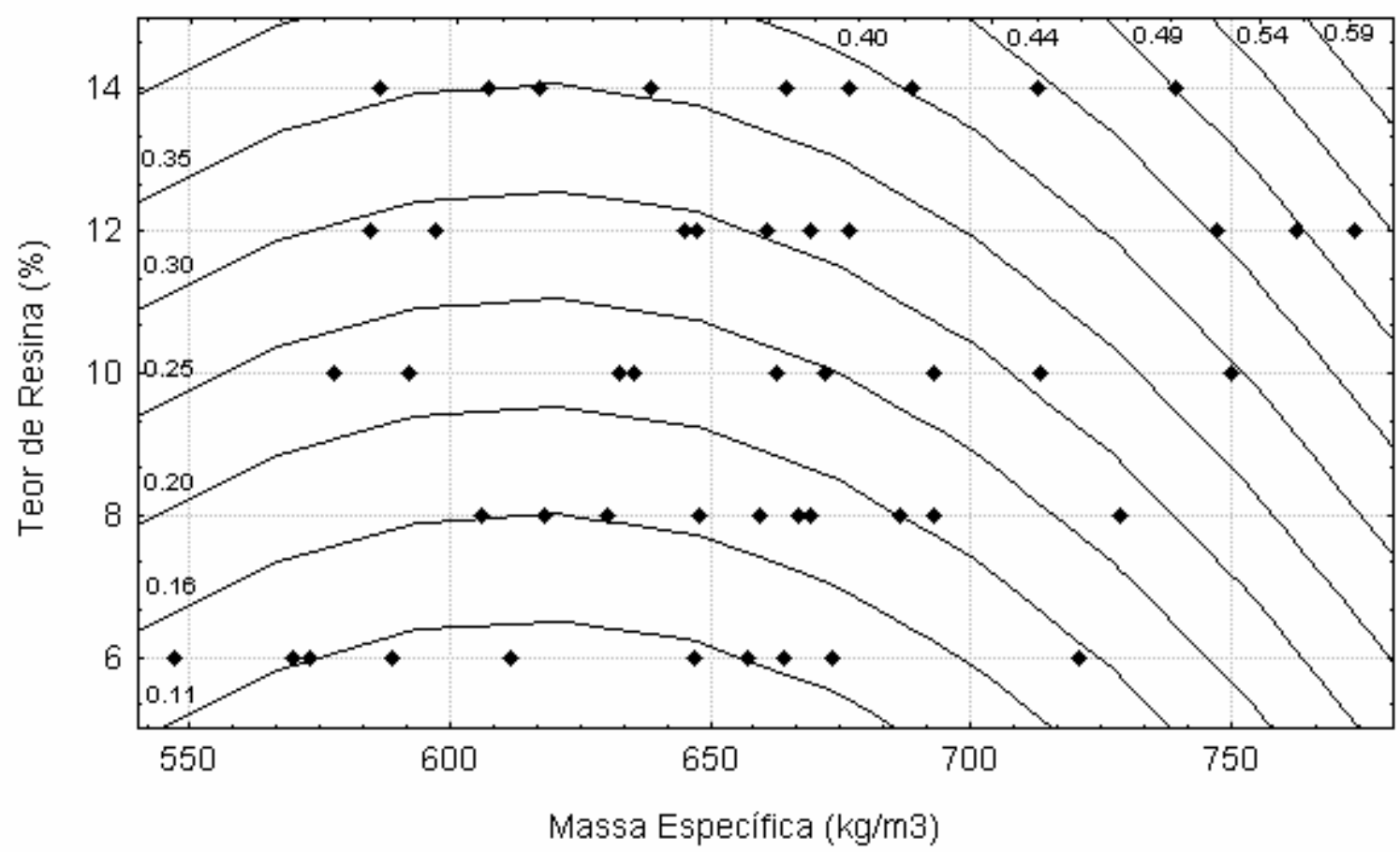

FIGURA 7: Isolinhas de adesão interna (MPa) em razão da massa específica $(\mathrm{kg} / \mathrm{m} 3)$ e do teor de resina (\%) dos painéis MDF, com os losângulos representando os pontos obtidos experimentalmente.

\section{CONCLUSÕES}

Com exceção do módulo de elasticidade, todas as variáveis apresentaram maior correlação com o teor de resina do que com a massa específica.

Todos os modelos obtidos, para determinar as variáveis dependentes, incorporam, de maneira significativa, os fatores teor de resina e massa específica.

A interação entre massa específica e teor de resina só foi significativa a $1 \%$ para inchamento em espessura.

O módulo de ruptura, de elasticidade e a adesão interna apresentam comportamento diretamente proporcional ao teor de resina e à massa específica.

O inchamento em espessura e a absorção de água apresentam comportamento inversamente proporcional a essas variáveis. 


\section{REFERÊNCIAS BIBLIOGRÁFICAS}

BENADUCE, C. Fabricação de Painéis de Média Densidade (MDF) a partir de Fibras de Eucalyptus grandis W. Hill Ex Maiden e Pinus caribaea Morelet var. hondurensis Barret e Golfari. 1998. Dissertação (Mestrado em ...) - Escola Superior de Agricultura "Luiz de Queiroz", Universidade de São Paulo, Piracicaba.

CARLL, C. G. Review of thickness swell in hardboard siding: effect of processing variables. Madison, WI: USDA, Forest Service, Forest Products Laboratory, 1996. 10 p. (Gen. Tech. Rep. FPL-GTR-96.)

CHOW, P. Properties of medium-density, dry-formed fiberboard from seven hardwood residues and bark. Forest Products Journal, v. 26, n. 5, p. 48-55, May 1976.

EUROPEAN COMMITTEE FOR STANDARDIZATION. European Standard EN 319 particleboards and Fiberboards - determination of tensile strength perpendicular to the plane of the board. Bruxelas, 1993.

EUROPEAN COMMITTEE FOR STANDARDIZATION. European Standard EN 310 - woodbased panels - determination of modulus of elasticity in bending and of bending strength. Bruxelas, 1993.

EUROPEAN COMMITTEE FOR STANDARDIZATION. European Standard EN 317 particleboards and Fiberboards - determination of swelling in thickness after immersion in water. Bruxelas, 1993.

EUROPEAN COMMITTEE FOR STANDARDIZATION. European Standard EN 323 - woodbased panels - determination of density. Bruxelas, 1993.

KELLY, M. W. Critical literature review of relationships between processing parameters and physical properties of particleboard. Madison, WI: USDA, Forest Service, Forest Products Laboratory, 1977. 65 p. (Gen. Tech. Rep. FPL-10).

LABOSKY Jr., P.; YOBP, R. D.; JANOWIAK, J. J. et al. Effect of steam pressure refining and resin levels on the properties of UF-bonded red maple MDF. Forest Products Journal, v. 43, n. 11/12, p. 82-88, Nov./Dec. 1993.

MALONEY, T. M. The family of wood composite materials. Forest Products Journal, v. 46, n. 2, p. 19-26, Feb. 1996.

MALONEY, T. M. Modern particleboard \& dry-process fiberboard manufacturing. San Francisco: Miller Freeman, 1989. 672 p.

NELSON, N. D. Effects of wood and pulp properties on medium-density, dry-formed hardboard. Forest Products Journal, v. 23, n. 9, p. 72-80, Sep. 1973.

PIZZI, A. Advanced wood adhesives technology. New York: Marcel Dekker, 1994. 297 p.

PUGEL, A. D.; PRICE, E. W.; HSE, C. Y. Composites from southern pine juvenile wood. Part 1. Panel fabrication and inicial properties. Forest Products Journal, v. 40, n. 1, p. 29-33, Jan.

Ciência Florestal, v. 10 , n. 2, 2000 
1990.

SUCHSLAND, O.; WOODSON, G. E. Fiberboard manufacturing practices in United States. Washington, DC:USDA, 1987. 263 p. (USDA. Agricultural Handbook n. 640)

WOODSON, G. E. Properties of medium-density fiberboard related to hardwood specific gravity. In: TENTH PARTICLEBOARD SYMPOSIUM, 1976, Washington. Proceedings... Washington: Washington State University, 1976. p. 175-192. /Resumo publicado no CAB Abstracts on CDROM/ 